\title{
Mental Health Act
}

\section{The rise in the use of the MHA to detain people in} England

\section{Contents}

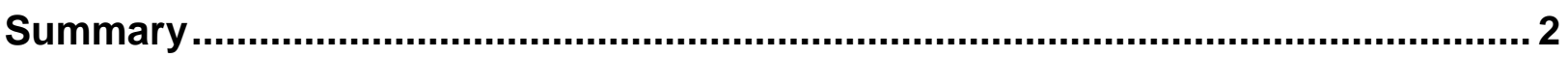

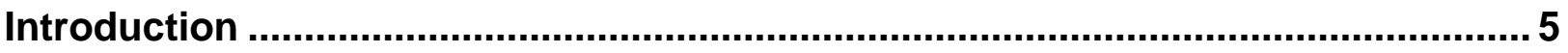

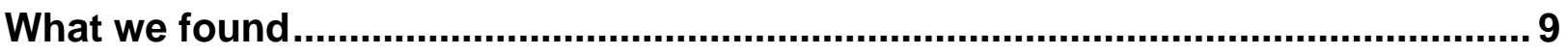

1. The impact of changes in mental health service provision, bed management and

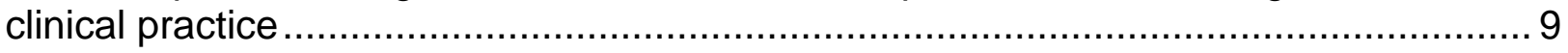

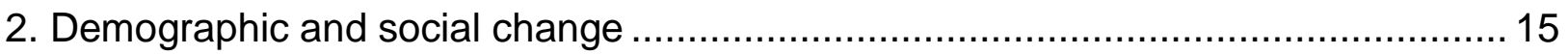

3. Legal and policy developments that have influenced practice.......................... 19

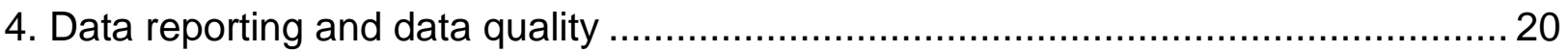

What local learning can help national programmes reduce the use of the Mental Health Act? ................................................................................................................ 22

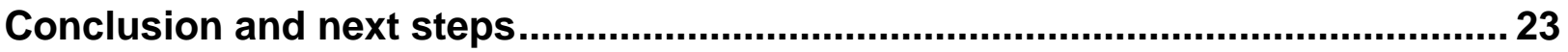

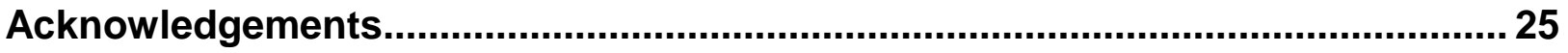

Appendix: The Mental Health Act and other legal frameworks.......................... 26

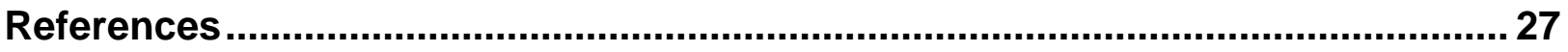




\section{Summary}

Between 2005/06 and 2015/16, the reported number of uses of the Mental Health Act (MHA) increased by $40 \%$. It is well established that people from Black and minority ethnic groups are much more likely to be detained than those in White British groups. ${ }^{1}$ In October 2017, in response to these and other concerns, the government announced an independent review of the MHA. This review will make recommendations for improvements to legislation and practice in late 2018.

In 2016, CQC committed to working with local services to gather views on the reasons for the national increase in the use of the MHA. During 2017, we visited eight NHS trusts, two independent mental health service providers and 23 local authorities to explore the factors that might account for the increasing use of the MHA. The sites included parts of the country where rates have fallen or remained the same over the previous three years, as well as areas where there has been an increase. On these visits, we met with more than 60 detained patients, 30 carers and more than 250 staff.

Although we cannot be certain about their relative impact, we identified a number of factors that might influence the rates of detention and grouped them into four broad themes:

\section{Changes in mental health service provision and bed management}

More frequent readmissions - either as part of a plan of care or because of premature or poorly planned discharge.

Loss of specialist community teams offering alternatives to admission.

Presure on beds preventing early, informal admissions or leading to discharge without adequate support to stay well once out of hospital being in place.

3. Legal and policy developments that have influenced practice

2007 reform of MHA widening definition of mental disorder and of treatment.

Increasing awareness of the factors of de facto detention as a result of the Bournewood judgement, and Mental Capacity Act led to more use of MHA for those lacking capacity.

Greater police awareness of mental disorder leading to more diversions from the criminal justice system.

\section{Demographic and social change}

Increase in population size and in sections of the population 'at risk' of detention (especially older people with dementia).

Rising inequality and social exclusion (for example rising homelessness).

Impact of alcohol and substance misuse.

\section{Data reporting and data quality}

Improved completeness of local returns that inform national data sets.

Duplicate entries - including doublecounting when a detained patient is moved from one ward or provider to another. 


\section{Our hypotheses for the rising rate of detention}

We did not expect this qualitative work to identify all of the detailed factors that might cause the rising rate of detention. We also knew that it would not allow us to make confident statements about the extent to which each of the factors have contributed to the rise. However, based on the four themes, we developed eight hypotheses that we think are likely to cover all factors that might have made some contribution to the rise in use of the MHA:

1. The apparent rise in rate of detention since 2010 is in part due to the national data return being more complete or to an increase in duplicate returns.

2. More people are being detained on more than one occasion during a calendar year than was previously the case.

3. As bed numbers have fallen, more people with severe mental health problems are living outside of a hospital setting and so are at greater risk of being detained.

4. Some people are being detained under the MHA who would previously not have been detained. This is because clinicians are applying the criteria for detention differently to people with certain types of disorder (such as dementia or personality disorder). It could also be because more people with mental health problems are coming to the attention of mental health care workers (for example, through schemes that divert people from the criminal justice system).

5. People who need admission and who would previously have agreed to informal admission are now refusing and are being admitted as detained patients.

6. Admissions (some of which would be formal) that could in the past have been prevented are now not being prevented because less restrictive alternatives in the community are not available.

7. There has been an increase in the total size of the population of England and an increase in the size of those sections of the population that are more at risk of detention.

8. There has been an increase in the prevalence of risk factors for detention, such as social exclusion and problematic, untreated drug and alcohol misuse. 
We can be confident about a number of our conclusions:

- There is no single cause for the rise in rates of detention this decade. It is highly likely that a range of factors are at play both nationally and locally.

- The rise in part suggests a system under considerable pressure. Staff in some areas have limited access to community services that can act as true alternatives to admission. At the same time, these services may have no bed available for an admission when it is needed. This creates a dilemma for both patient and staff, and reduces the likelihood that detention can be avoided - either by providing a less restrictive community alternative or by an informal admission to prevent further deterioration. In some places, this might amount to a vicious cycle where pressure on beds leads to clinical practices that increase the likelihood of patients being detained, which itself increases the pressure on beds.

- We found no evidence that professionals are using the Mental Health Act to admit people who do not meet the criteria for detention, for example to 'game' the system to obtain a bed for the patient.

- We think it is unlikely that reform of mental health legislation on its own will reduce the rate of detention. There must also be action to address the underlying problems that almost certainly contribute to the rise this decade.

- The pattern of change in rates of detention over the past 25 years (figure 1, page 5) supports our conclusion that changes in mental health law need to happen alongside action to address wider problems. Following year-on-year increases during the 1990s, the rate of detention fell between 2000 and 2009 before starting to rise again. The mental health National Service Framework was published in $1999 .^{2}$ The decade that followed was a time of significant investment in new community teams, whose purpose was to act as alternatives to hospital admission. 


\section{Introduction}

National data from the last 25 years shows an increasing use of the Mental Health Act (MHA) to treat people in hospitals. Figure 1 shows how many times the MHA has been used, but not how many people are being detained. This is because it does not tell us whether the same person has been detained more than once in the same period. This is the case for the data set used up to but not including 2016/17. It also does not tell us what happened to the patient before or after their hospital admission, nor their race, any other protected characteristic, age, diagnosis, housing situation or economic status.

Between 2005/06 and 2015/16, the reported number of uses of the MHA to detain people in hospital increased by $40 \%$. There was a $9 \%$ increase between $2014 / 15$ to $2015 / 16$ rising to 63,622 uses of the MHA.

Figure 1: Detentions in hospital under the MHA, 1990/91 to 2015/16

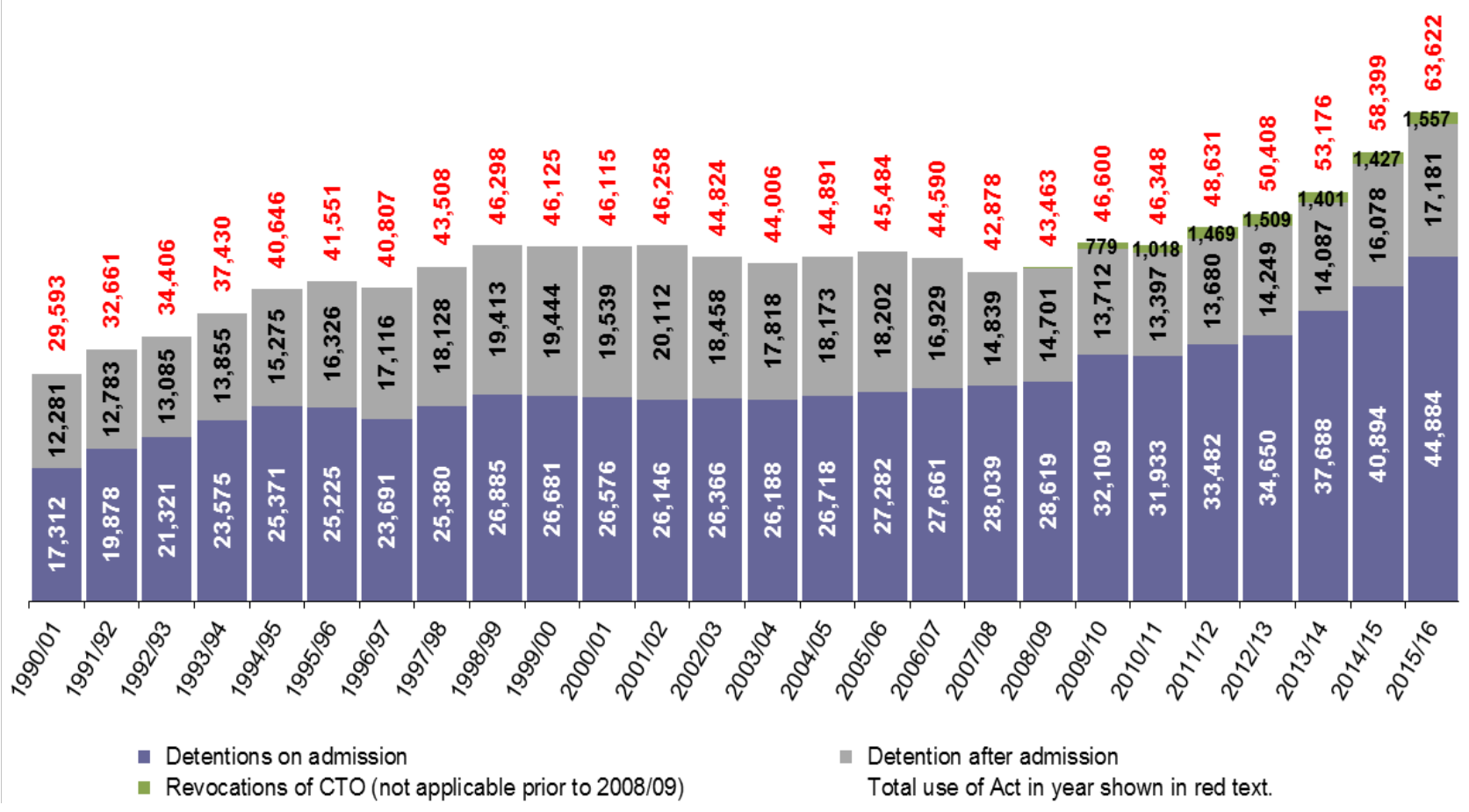

Data source: NHS Digital, KP90 / Mental Health Services Data Set (MHSDS)

Figure 1 shows that, in marked contrast to substantial rises in the 1990s and since 2010 , there was a steady reduction in the number of detentions between 2000 and 2009. Below we speculate that this might have been due to the introduction of specialist community mental health teams following publication of the National Service Framework for Mental Health in 1999. ${ }^{3}$

A separate source of data provides information about how many people are detained in hospital under the MHA on 31 March each year (figure 2). This shows a steady increase over the past 20 years in the number of people detained in hospital. On 31 March 2016, there were a total of 20,151 - the highest number recorded to date.

Although, as figure 2 shows, the number of people detained in hospital on 31 March increased substantially between 2010 and 2016, this was less than the increase (shown in figure 1) in the number of times the MHA has been used to admit a person or to detain them after admission ( $21 \%$ versus $37 \%$ ). This could be the result of more 
individuals being detained for a short period of time, or of an increase in the proportion of individuals who are detained more than once in the year, or a combination of both factors.

We do not see a dip in the size of the detained population in the 2000s that corresponds to the reduction in the number of uses of the MHA to detain people. This may be because the new community teams reduced the number of brief admissions or re-admissions but did not affect the underlying trend in the rise in people requiring longer term admissions as detained patients. Another possible reason could be that concerns over de facto detention in the early 2000s as a result of the Bournewood case (1998 onwards) ${ }^{4}$ and the introduction of the Mental Capacity Act (MCA) in 2005. This led to some services reporting increases in the number of their longer stay patients being made subject to the MHA, who may have been admitted informally previously but did not have the capacity to consent their admission.

Figure 2: Detained patient population at 31 March, 1997 to 2016

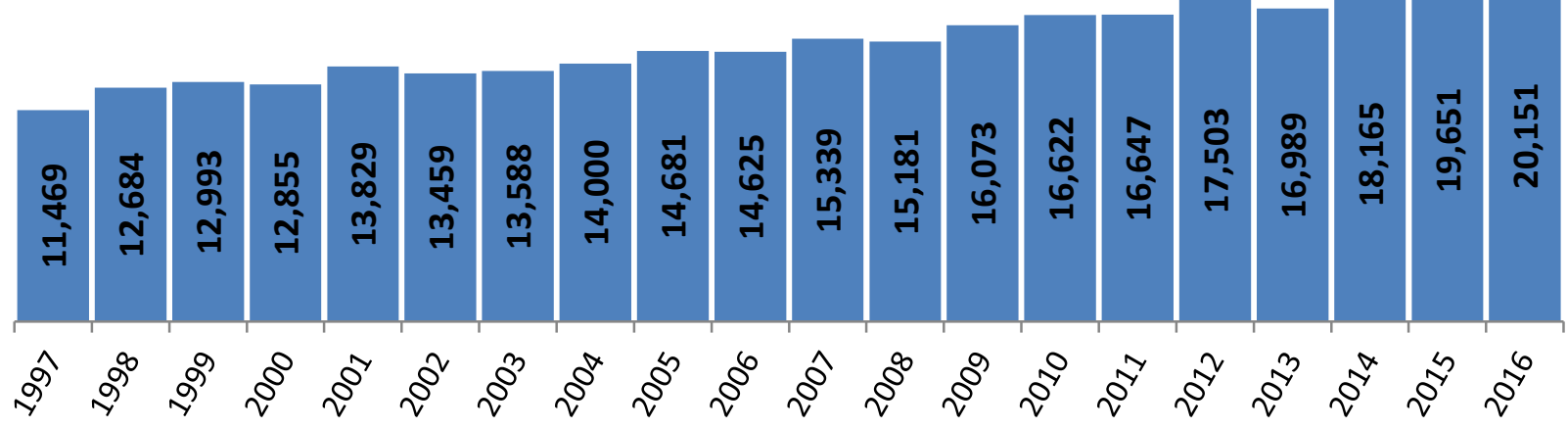

Data source: NHS Digital, KP90

The reported rise in the number of detentions, and in the number of people detained in hospital, has happened during a period when the number of mental health beds has been greatly reduced (figure 3 ). Although we found no causal link in the providers we visited and we believe it is likely that where there are effective plans to provide alternatives to admission, bed reductions would not necessarily lead to increased detentions under the Act. In the previous 20 years, the number of beds has more than halved but the rate of detention has increased by about $50 \%$, and the number of people detained in hospital at any one time has risen by $75 \%$. The inevitable result is that a far higher proportion of people in mental health wards are detained under the MHA than was the case in the past. We are not aware of any evidence to suggest that the reduction in bed numbers is itself a direct cause of the rise in rates of detention. This should not be the case if bed closures have been accompanied by the development of community mental health services that are real alternatives to admission. However, it is possible that high rates of detention may be a feature in areas that have both low bed numbers and challenges in providing effective alternatives to admission. 


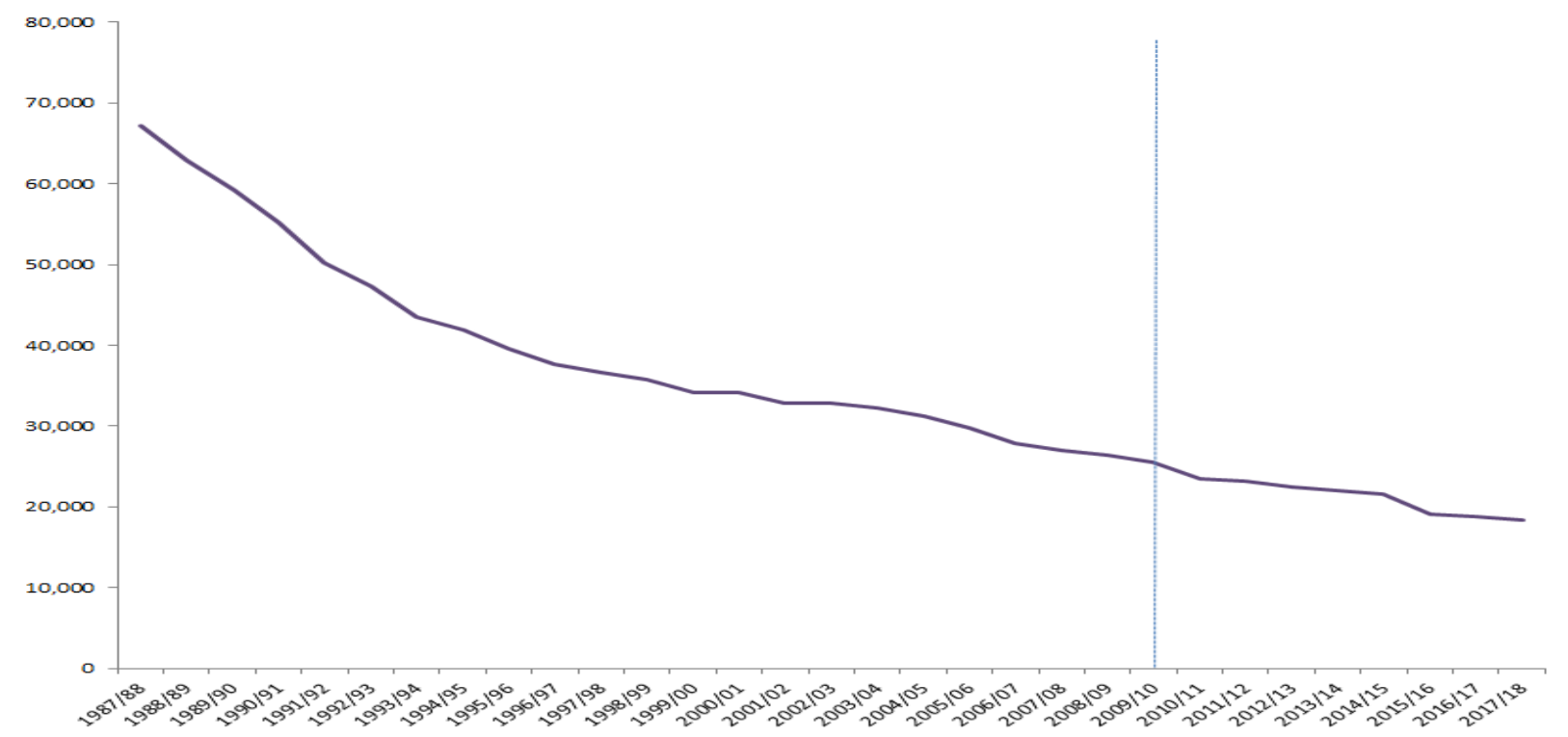

Data source: NHS England bed availability and occupancy data - overnight.

Note: The data set changed between 2009/10 and 2010/11 and moved to a quarterly collection period. Data from 2010/11 onwards has been averaged across the available quarters in the year. This means that the data may not be directly comparable with previous years.

The Five Year Forward View for Mental Health, published in 2016, sets out recommendations for national bodies to improve mental health services and achieve the ambition of parity of esteem between mental and physical health for everyone. It specifically includes reducing MHA detentions, tackling the inequalities of overrepresentation of people from Black and minority ethnic groups (BME) and other disadvantaged groups of people, and improvements to access to good quality mental health care. ${ }^{5}$ The Commission set up in 2015 to review the provision of acute inpatient psychiatric care for adults, led by Lord Crisp, also highlighted areas that have an impact on the quality of care provided to people subject to the MHA. ${ }^{6}$ The Commission recommended that local service capacity assessments and improvement programmes should take place, and that providers and commissioners should ensure that there is appropriate inpatient and community-based acute provision, including acute beds and resources in crisis resolution and home treatment teams, to meet the needs of patients. It also noted the importance of whole system capacity management to ensure that pathways of care work effectively. This means that beds should be available when they are needed, as one part of a well-functioning local acute mental health system.

In October 2017, the government announced an independent review of the MHA. Concern about the rising use of the MHA, and the over-representation of people from BME communities in the detained population, were factors that contributed to this decision. The MHA review comes at a time of unprecedented challenge for the mental health sector, as outlined in our report, The State of Care in Mental Health Services 2014 to $2017^{7}$

In 2016, CQC committed to working with local services to gather views on the reasons for the national increase in the use of the MHA, to identify how local services are responding to the changing activity, and to help inform the targeted work planned to bring about improvement. This report presents our findings. 


\section{Our methodology}

The main purpose of this review was to bring the perspective of patients, carers and staff in local services to the national debate about the causes of the rise in rates of detention. This report is based on information from three sources:

- Data review: We analysed available data on the use of the MHA nationally. We also asked sites that we visited to send us information from their local systems to help identify how the MHA was being used, how they kept this under review and what initiatives were in place to respond to changes in activity. This information was available to all site visit teams.

- Site visits: During 2017, we visited eight NHS trusts, two independent mental health service providers and 23 local authorities to explore the factors that affect the use of the MHA. The sites included parts of the country where rates had fallen or remained the same over the previous three years, as well as areas where there had been an increase. On these visits, we interviewed or held focus groups with more than 60 detained patients, 30 carers and more than 250 staff. The interviews and focus groups were open and informal to encourage participants to contribute to the national debate by exploring factors at play locally.

- Engagement: throughout the review, we shared and tested the emerging findings with our MHA External Advisory Group, Service User Reference Panel and other stakeholders (see appendix).

\section{Patients' views on the rising use of the MHA}

During a focus group, patients told us their views on the rising use of the MHA based on their experiences of detention. They felt that spikes in detention are a reflection of several factors. Some of the examples are below:

- A culture based on the level of risk the person poses to themselves and others, rather than a culture that focuses on their recovery.

- Despite the crisis teams being excellent at supporting and proactively preventing crisis, while a crisis was urgently escalating they were not responsive enough. Although a crisis telephone helpline is $24 / 7$, practical face-to-face help is not available 24/7.

- Patients generally felt there were not enough beds and said that they had experience of not being detained due to a lack of beds. 


\section{What we found}

When presenting our findings and conclusions, we do not start from the assumption that a rise in the rate of detention is necessarily a bad thing. It might, for example, reflect that more people who would benefit from mental health care are now receiving it; or that some people who might previously have been held on a mental health ward with no lawful authority ('de facto detention') may now be detained under the MHA and so subject to the safeguards it provides.

\section{The impact of changes in mental health service provision, bed management and clinical practice}

\section{The configuration and function of community mental health teams and the availability of alternatives to admission}

Patients, carers and staff - from both providers and commissioners - agreed that a greater focus on early intervention and intensive support in the community had the greatest potential to reduce admissions to hospital and likelihood of using the MHA.

This is consistent with the Crisp Commission; which was set up in response to widespread concerns about the provision of acute inpatient psychiatric beds and alternatives to admission available for patients. The Commission reported that many people felt that there is a relationship between the strength of community services and reduced pressure on inpatient services and fewer out-of-area placements. The Commission was also told that the better community services are integrated into the whole system, the better their collective ability was likely to be in preventing hospital admission in the first place and discharging patients more quickly. ${ }^{8}$

In some areas, those we interviewed told us that specialist community mental health teams, capable of providing intensive support for people with complex needs, had been decommissioned or merged to form teams with more generic functions. These included assertive outreach and home treatment teams. This has led to reduced capacity to make frequent, low-level interventions, or visits that might support a person whose condition has deteriorated. Also, staff in more generic teams tended to have higher caseload sizes and so could not provide more intensive support to individuals. The change had also led to reduced continuity in the care of people with long-term problems. The specialist teams that work with people with complex needs are able to form longer term relationships with patients who are vulnerable to relapse. This tends to make staff more prepared to take positive risks by, for example, not admitting the patient to hospital during periods of relapse.

As mentioned above, the long-term pattern in rates of detention supports the suggestion that the loss of these specialist teams might have contributed to a rise in rates of detention in some areas. The reduction in rates of detention between 2000 and 2009 (figure 1, page 5) coincided with a period when NHS trusts established teams to provide early intervention in psychosis, assertive community treatment and crisis resolution and home treatment. The rising rate of detention seen in recent years could reflect the end of the National Service Framework mandates to invest in such specialised local services and teams. However, the recent establishment of NHS England's mandated national access standard for Early Intervention in Psychosis and new national investment in crisis resolution home treatment teams (CRHTTs) 
to provide a genuine alternative to admission (in line with their intended purpose), could have a positive impact to reverse these trends again.

During our visits, we found examples of local initiatives that recognised the important role of community services and alternatives to hospital admission. This included a commitment of $£ 7$ million in additional investment by one clinical commissioning group (CCG) to reduce out-of-area placements and improve the bed occupancy for mental health wards. The providers that we visited that had retained specialised teams were convinced of their value. NHS England and NHS Improvement's major national programme to support local areas to eliminate non-specialist out-of-area placements (OAPs) by 2021 could therefore also lead to a positive impact in this regard.

\section{Early identification of deterioration in mental health}

Birmingham and Solihull Mental Health NHS Foundation Trust suggested that effective community-based health and social care services were able to reduce the need to detain patients. These teams (including the home treatment teams, assertive outreach and community mental health teams) were able to identify deterioration in mental health early and intervene before detention became necessary. People at the trust thought that the home treatment teams had a significant impact on the care and treatment of patients in the area. They were seen as effective and successful in helping people who use mental health services to stay in their communities.

\section{Working with home treatment teams}

We were told that well-resourced home treatment teams have had an impact on the detention rates in Cornwall Partnership NHS Foundation Trust. The local police chief inspector provided examples of where they had changed police actions following consultation with the home treatment team, using their knowledge of patients. The manager of the home treatment team was responsible for an alternative provision fund, backed by the clinical commissioning group. The fund was designed to provide extra support in someone's own home to avoid unnecessary admission or reduce the length of time they were admitted. The service also developed positive behaviour support plans with a number of patients. These were good examples of contingency and forward planning and helped to reduce the need for detention. The trust managed to carry this out despite reporting fewer acute inpatient beds than trusts in other parts of the country.

People from all the services we visited flagged their concerns about the inadequacy of other services that might divert people from being admitted to hospital. This included a lack of crisis houses. When such services were available, people sometimes described long waiting lists or delays in funding for individual patients. This problem with access to help when it is needed urgently is a theme in the Five Year Forward View for Mental Health, the report of the Crisp Commission and the work of the Crisis Care Concordat. $^{9,10,11}$ The 2017 Community Mental Health Survey found that, of those respondents who knew who to contact in a crisis, and tried to do so, only $39 \%$ said they 'definitely' got the help they needed. ${ }^{12}$ This was worse than in 2014 , when $45 \%$ said that they definitely got help. The national joint NHS England and NHS Improvement 
Out of Area Placement programme is clear that reductions will only be achievable by taking a whole system view and strengthening out of hospital care e.g. community, crisis houses, CRHTTs, crisis lines.

\section{The impact of the low number of beds and pressure on mental health wards}

As figures 1 to 3 show (pages 5 to 6), since 2010, the number of mental health beds has fallen but the rate of detention, and the number of people detained in hospital at any one time, appear to have both risen. Inevitably, this has put pressure on beds, raised the threshold for admission and means that the proportion of inpatients who are detained under the Mental Health Act is high and increasing. Some NHS wards will only admit people subject to the MHA and we were told that access to independent hospitals could only be secured if the patient is detained. On one site, we heard that bed occupancy was often above $100 \%$. This meant that even patients requiring admission under section 2 of the MHA might experience a delay in being admitted.

We were told that delays in admission due to a bed not being available may mean that a patient, who might have consented to be admitted informally at an earlier stage, may deteriorate and become unwilling or unable to agree to an admission, and therefore need to be detained under the MHA. This problem could be lessened by close joint working between community and inpatient mental health teams, so that patients are prioritised based on a joint assessment of clinical need. NHS England have stated the focus on reducing out of area placements by strengthening community-based acute provision and improving overall system capacity management and flow should result in more timely access to local inpatient care when it is needed.

\section{Reviewing patterns of admission}

In Sussex Partnership NHS Foundation Trust, approved mental health professionals (AMHPs) and doctors raised concerns over how difficult it was to secure a bed for informal patients. The AMHPs and doctors felt that this discouraged staff who cared for patients to apply the least restriction principle. The trust's MHA Monitoring Group agreed that its AMHP leads would start a three-month programme of monitoring how difficult it was to secure a bed for informal patients. The programme would look at whether the difficulty led to patients being detained under the MHA. If an informal patient was then detained due to not being able to secure a bed, the trust agreed to raise it as a serious incident.

As well as making it more likely that the people with greatest need are prioritised for admission, good bed management can also enable better discharge management. Staff in some services reported difficulties in granting patients overnight trial leave before discharge, because bed managers expect patients who can spend a night out of hospital to be discharged. Many people we spoke to felt the risk of repeat detentions for the same person will increase if they have to be discharged without trial leave. 


\section{Monitoring and supporting discharge arrangements}

Birmingham and Solihull Mental Health NHS Foundation Trust carried out daily reviews of patients who were on the waiting list to be admitted. Staff considered the risks and contingency plans for each patient. The trust also carried out a weekly bed management review every Tuesday to look at possible discharges and barriers to discharge. These often included problems in finding social care placements or funding.

The trust piloted the 'Red to Green' system - an idea that originated in acute general hospital settings to monitor whether scheduled interventions had been achieved on time and that each day spent in a hospital setting was value-adding. ${ }^{13}$ On a mental health ward, this might mean making arrangements to make sure that the relevant people attend a Care Programme Approach or section 117 aftercare meeting. The system guarantees that meetings can be a meaningful forum to move patient care and discharge plans forward.

In our report, The State of Care in Mental Health Services 2014 to 2017, we describe mental health admission wards as a "high risk environment" with a high number of incidents of assaults on patients and staff. ${ }^{14}$ We also reported that "some services struggle to ensure that mental health wards are staffed safely at all times". The resulting impact on the ward environment, coupled with the high likelihood in some areas that admission might be to a bed a long way from home, might reduce patients' willingness to accept admission when they are offered it. Staff in one service told us that the smoking ban increased the likelihood of patients being detained under the MHA. This is because some patients, who would usually agree to be informally admitted or stay in hospital, refuse because they are unwilling to give up smoking while admitted.

During our visits, no staff that we interviewed told us that they would detain a person under the MHA solely as a means to get access to a bed. This is in contrast to findings from a survey of psychiatric trainees when 104 of 576 surveyed said their decision to detain a patient would be influenced by the fact that doing so may make the provision of a bed more likely. ${ }^{15}$ Staff told us that they would only consider detention if the patient needed treatment or assessment and that admission was needed to protect their health and safety or that of others. We did hear from groups of professionals who felt the use of the MHA would mean it is more likely to get a bed, which is supported by the other findings in this report.

If mental health care workers were detaining people who did not met the criteria for detention, simply to obtain a bed; we would expect to see a rise in the number of successful appeals against detention. Data from the First-tier Tribunal (Mental Health) show no such increase. The percentage of appeals leading to absolute discharge from detention has remained at around $5 \%$ over many years (figure 4 ). The rate at which doctors discharge patients before their tribunal hearing takes place (shown as 'total discharged before hearing') has altered over the last years, but has not risen disproportionately to the rising number of Tribunal applications. 
Figure 4: Applications, hearings and discharges: the First-tier Tribunal (Mental Health), 2010/11 to 2016/17

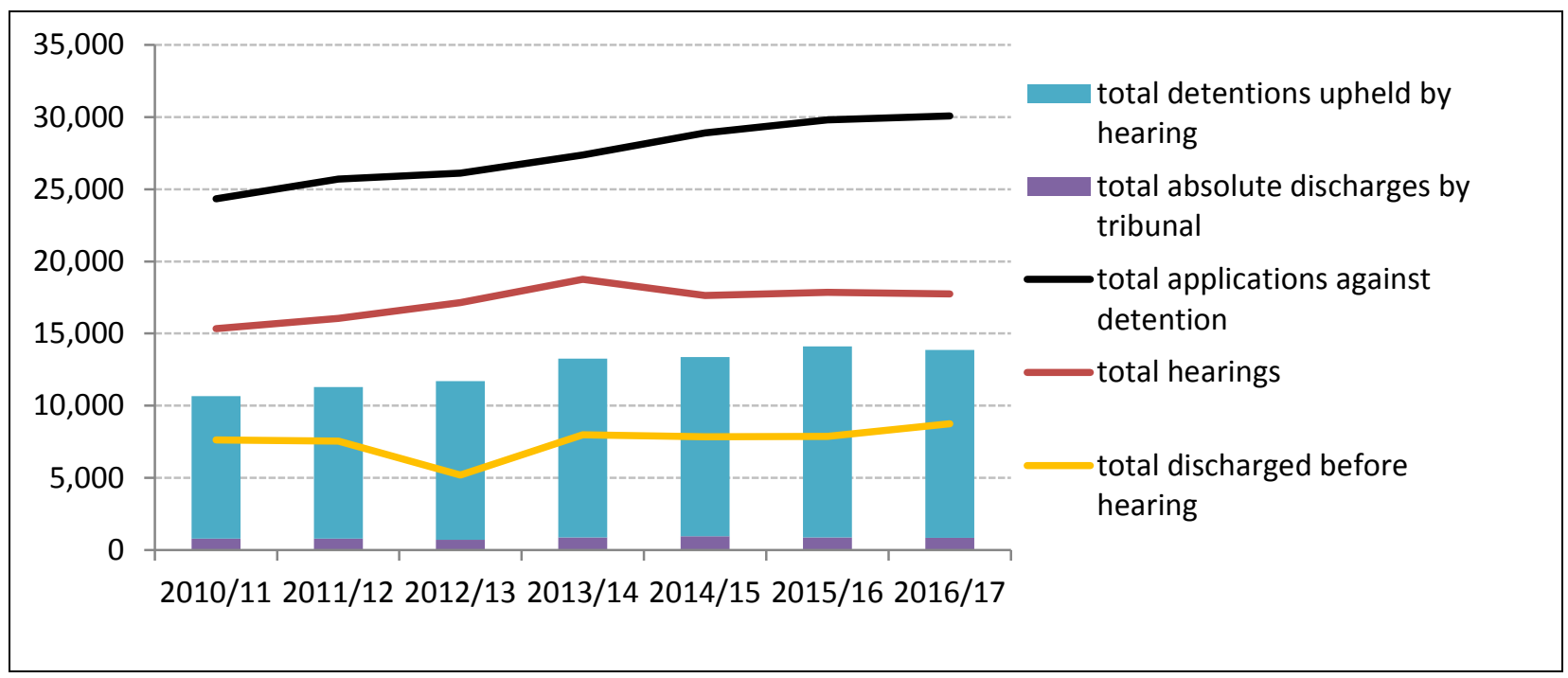

Data source: First Tier Tribunal (Mental Health).

\section{The potential impact of readmissions}

As described above, there are two ways to count how often the MHA is used to detain people. The first is to count how frequently the MHA is used over the course of a year to admit a person to hospital or to detain them after they have been admitted (figure 1, page 5). The second is to count the number of people who are detained in hospital on a particular day (figure 2, page 6). The year-to-year increase in the number of people detained in hospital on 31 March suggests that the total number of people who are subject to the MHA has increased. However, the number of people detained in hospital on 31 March has increased less than the number of times the MHA has been used to admit a person or to detain them after admission (21\% versus 37\% between 2010 and 2016).

There are a number of possible explanations for this. One is that more individuals are being admitted on a section of the MHA twice in one year than was the case in the past. Unfortunately, national data returns from the KP90 data set used as the official source of MHA data until 2015/16 have not allowed us to examine what contribution this makes to the rise in detention rates - although the change to the Mental Health Services Data Set as the official source of MHA data now makes this sort of analysis possible.

Some of the professionals that we interviewed thought it likely that some of the increase in uses of the MHA reflect a trend towards repeated, short periods of detention for treatment for some patients, who in the past might have experienced fewer but longer hospital stays. The people we spoke to thought this could be due to pressures on beds causing premature and inappropriate discharge. Ward managers told us of people who are admitted over and over again, in some cases due to difficulties in making thorough discharge arrangements. This is a particular challenge when a patient is not in the home borough and the ward team may not know local services so well. However, for some patients, repeated admissions might represent good practice and the less restrictive alternative to prolonged stays in hospital. 


\section{Monitoring readmissions to inform crisis planning}

South West London and St George's Mental Health NHS Trust monitored clinical data to identify all readmissions (defined as within 28 days of discharge) and made sure that a review took place. The trust looked in more detail at the pathway and clinical records for these patients.

For some patients who have frequent readmissions (especially those with a diagnosis of personality disorder), short cyclic admissions can be part of the appropriate care plan. Monitoring the readmission rates of detained patients allowed clinical teams to identify if the readmission was expected as part of a supportive care plan, or if they were due to difficulties related to discharge arrangements. Challenges were particularly great when the patient was not in the home borough and the ward team might not know local services so well.

\section{Using data to identify high-impact avoidable detention rates}

In 2013, the London region had the highest rates of detentions under the MHA, with twice the rate of inpatient detentions of other regions. NHS London established a pan-London multi-agency oversight and scrutiny committee and commissioned analysis of MHA and population data to help identify avoidable detention rates. Major variation between each local population area was identified, alongside potentially preventable causes of new and repeat detentions:

- Some of the variation could be accounted for by deprivation and associated high risk vulnerable populations that had not been prioritised for interventions and had no recourse to public funding.

- Using insights from British Transport Police analysis, a significant proportion of new detentions were due to mobility factors, for example, at major transport hubs such as airports and train stations bringing people into London from the rest of the UK and from abroad.

- Where public housing was limited, people were discharged into bed and breakfast accommodation with little support. This made them more likely to relapse and need re-detention and formal readmission. 


\section{Demographic and social change}

Some of the rise in rates of detention might be the result of local and national changes in the make-up of the population and in social factors that influence the use of the MHA.

\section{Changes in the make-up of the population}

The $37 \%$ rise in the number of detentions between 2010 and 2016 is far greater than the $5 \%$ increase in the total population of England over the same period (figure 5). However, it is possible that the impact of population changes might be greater than $5 \%$ because of disproportionate increases in the size of sections of the population that are more 'at risk' of detention - such as young men, some Black and minority ethnic (BME) groups and older people. In particular, the rise in the numbers of people living with dementia may have made a significant contribution to the rise in rates of detention.

Figure 5: Population increase versus rise in detention, 2010 to 2016

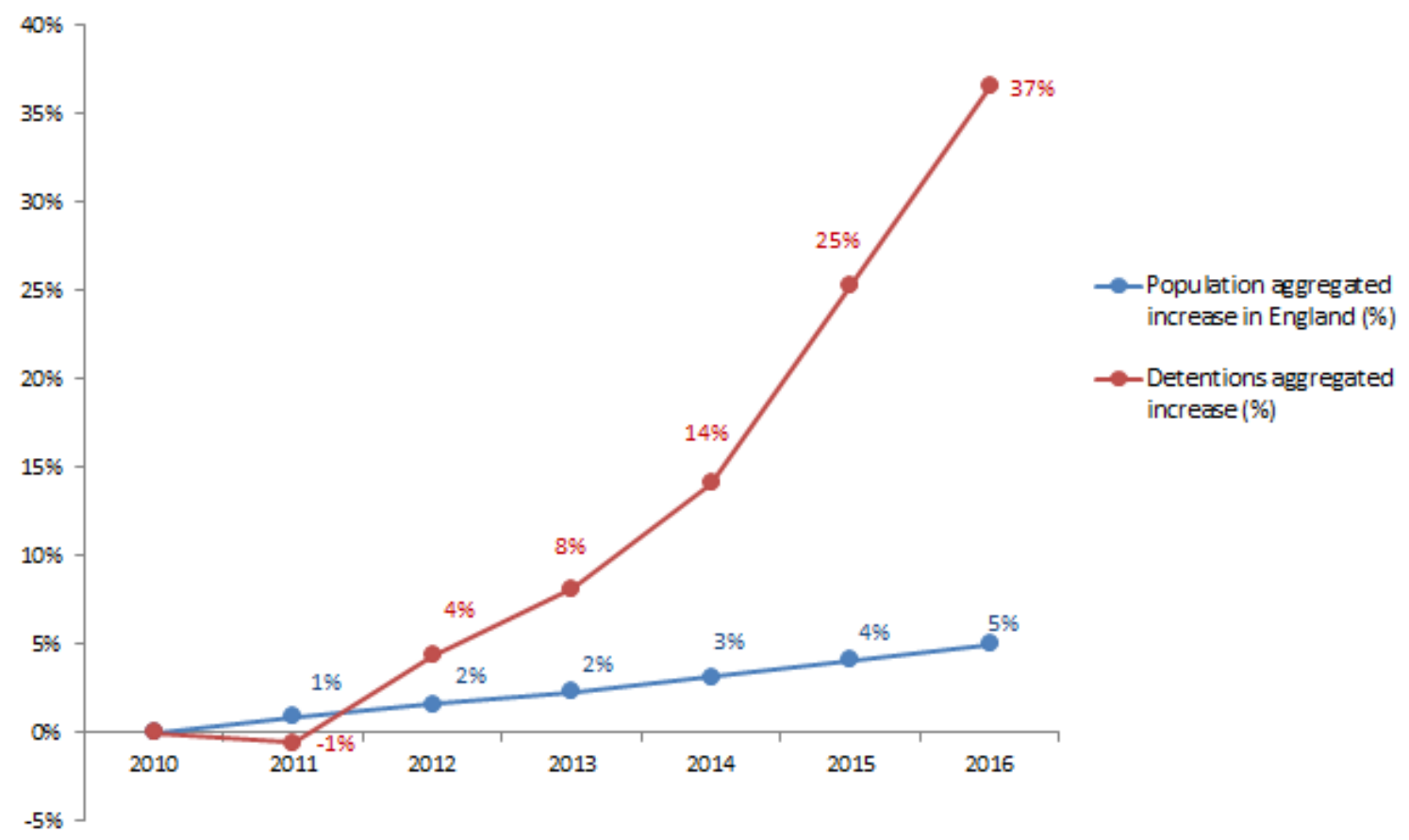

Data source: Detentions $=$ NHS Digital, KP90 and Population $=$ ONS

\section{The national over-representation of people from BME groups}

As figure 6 shows, we continue to see people from Black ethnic groups having much higher rates of detention than White people, nationally. The data in figure 6 represents a new collection for NHS Digital so comparison data from previous years is not available. However providers, researchers and national bodies believe this is a rising trend for BME groups. Many staff that we interviewed during our site visits recognised that people from BME groups - specifically Black Caribbean and Black African people - were over-represented in their local detained populations. It was commonly believed that Black people, and particularly Black men, will often have first contact with services late in their illness which makes them more likely to be detained. Some staff expressed the view that their services help to address this by becoming more accessible to BME communities. 
Figure 6: Detention rates by ethnicity, 2016/17

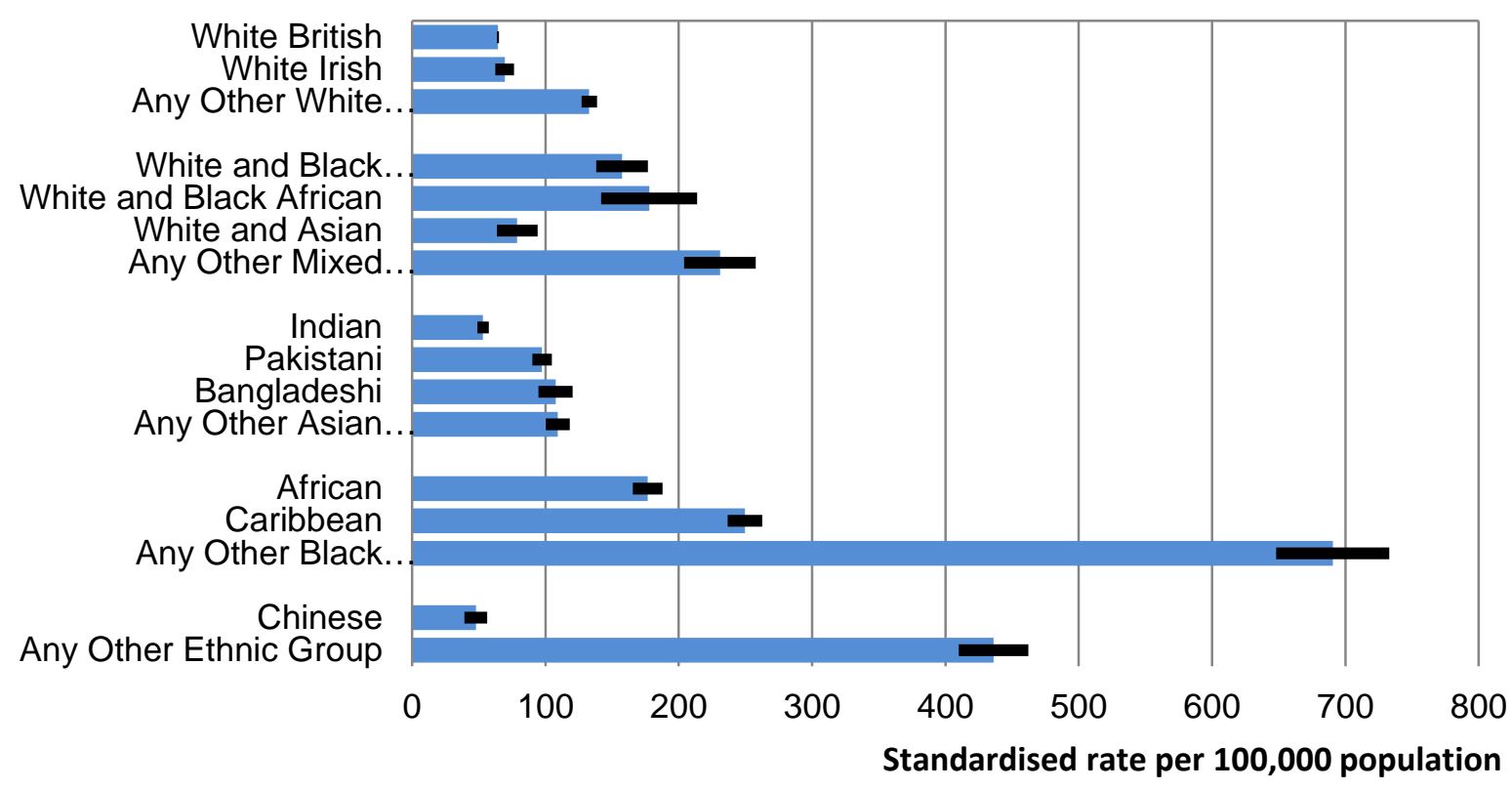

Source: NHS Digital

There are examples of BME communities developing educational initiatives on how to protect and prevent people from developing mental illness, reduce the stigma that adversely affects early intervention, and provide culture appropriate recovery services. One example is the Lambeth Black Thrive partnership.

\section{Reviewing rates of detention for people from BME groups}

East London Consortium of Clinical Commissioning Groups and East London NHS Foundation Trust have worked together, with input from clinicians, to develop a Commissioning for Quality and Innovation (CQUIN) project taking place over the next two years. The project will look at whether overall there are high rates of people from BME groups being detained in three of the boroughs covered by the provider and understand what could be done to reduce detentions.

In the first year, the project will focus on gathering existing and new data about overrepresentation and identify any shared characteristics of patients who receive multiple detentions. The project will then look at targeted interventions such as using crisis plans or increasing peer support.

This CQUIN will complement existing work with people from BME groups in secondary care, including initiatives to improve the experience of people who use services on wards. The City and Hackney BME psychology access service improves access to secondary care talking therapies for BME communities. It also has a wider commitment to use culturally sensitive techniques and approaches in other services such as Tree of Life groups, a therapeutic approach that values culture and spirituality as strengths and resources in the recovery process. 


\section{Engaging with people from BME communities}

In 2014, Birmingham and Solihull Mental Health NHS Foundation Trust worked on the '300 voices' project in partnership with West Midlands Police, Birmingham City Council and Time to Change. This project recognised the need to engage with young African and Caribbean men aged between 18 and 25 to hear their experiences of inpatient and outpatient care. This project was particularly important because young black men were over-represented in the figures for longer-term detentions and community treatment orders. Understanding their experiences may help services to reduce the number of detentions for this particular group.

\section{Rising inequality and social exclusion}

Staff in some areas believed that rising inequality, particularly in areas of relative deprivation, is a factor in the rise in detentions. They flagged issues with loneliness, poor housing options or homelessness, unemployment, poor physical health and family breakdown. The people we spoke with reported that the delivery of mental health care is becoming more complex overall, especially in responding to the needs of people who are homeless, rough sleepers, asylum seekers, immigrants, or those experiencing modern slavery. As well as it being more likely that people from these population groups will need mental health care, because of their transient state it is likely they may be unknown to local services and often difficult for health and social services to provide them with support before, during or after admission to prevent readmission through individualised management and personal care plans. This may increase the likelihood of them relapsing and being re-detained. National data shows an increase in homelessness applications (figure 7).

Figure 7: Homelessness applications and decisions by local authorities

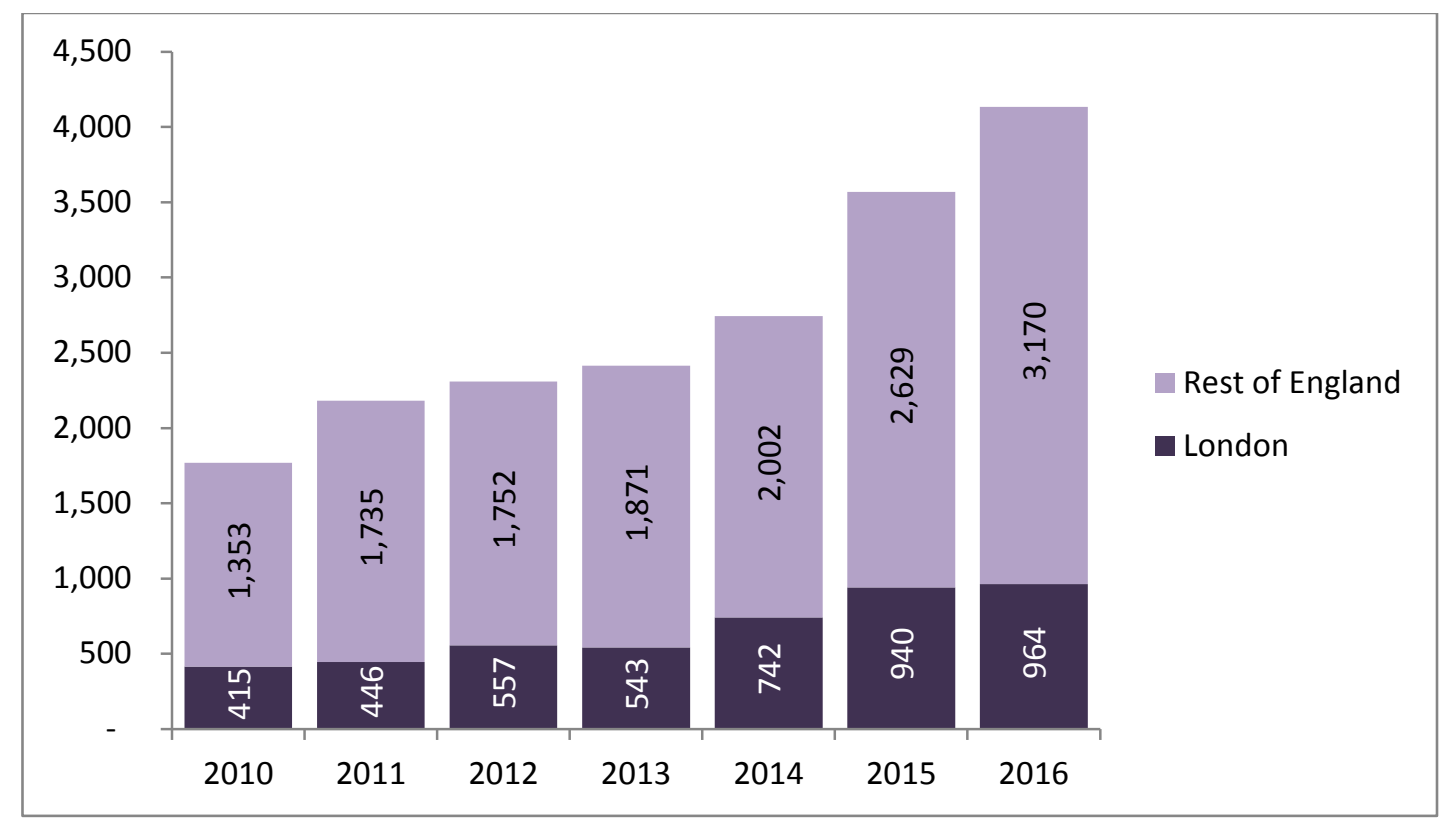

Source: Department for Communities and Local Government, Rough sleeping in England: autumn 2016 


\section{The impact of alcohol and drug misuse}

Although there has been an overall fall in the number of people using illegal drugs, the number of people who are admitted to hospitals with physical or mental health problems due to drug use is increasing. The number of hospital admissions with a primary or secondary diagnosis of drug-related mental health and behavioural disorders doubled between 2004/5 and 2014/15 (figure 8). In some areas, those we interviewed thought that alcohol and drug use, and particularly the use of synthetic cannabis substitutes such as 'spice', might have had an impact on the severity of people's mental health problems. This included reports of a greater likelihood of people being disinhibited or displaying behaviours that would lead to them being detained. A number of interviewees commented on this taking place during a time of cuts to drug and alcohol services, as a result of budget pressures in their local authorities.

Figure 8: Hospital admissions with a primary or secondary diagnosis of drugrelated mental and behavioural disorders, 2004/05 to 2014/15

\section{Thousands}

80

60

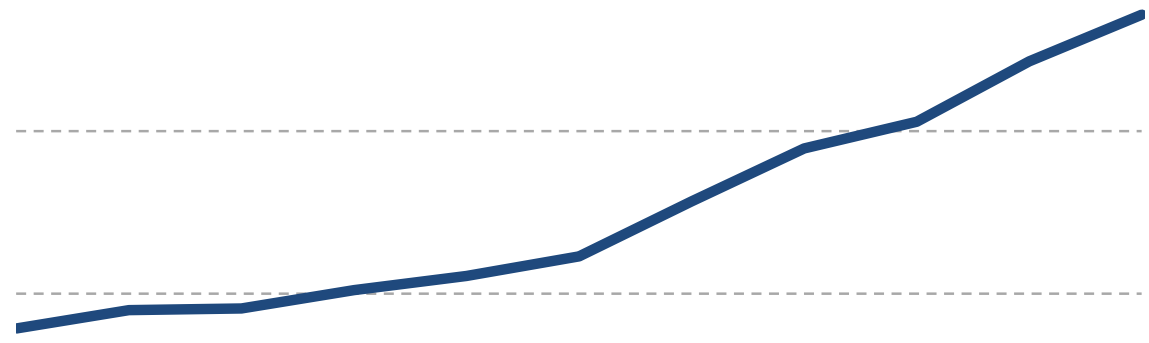

20

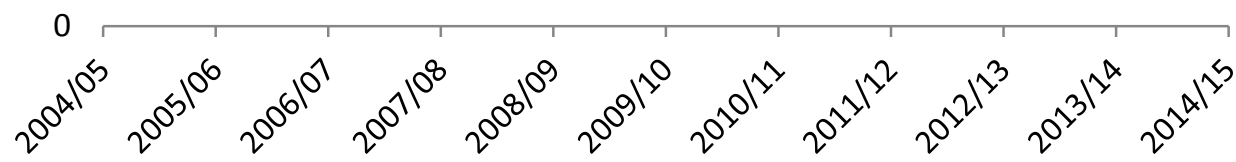

Source: Hospital Episode Statistics (HES) 2014/15

Note: The increase from 2004/05 will be partly due to improvements in recording of secondary diagnoses. 


\section{Legal and policy developments that have influenced practice}

Changes to mental health law and to policy guidance over the past decade may have contributed to the rise in rate of detentions as an unintended consequence.

\section{Changes to the MHA in 2007}

Staff in some services told us that they believed that they were more likely to have ongoing contact with people with certain diagnoses, such as borderline personality disorder, including as detained patients, than might have been the case in the past. They attributed this in part to a widening of the definition of mental disorder and the changes to the definition of 'treatment' that were made to the MHA in 2007.

\section{Increased recognition of patients that would benefit from the safeguards of the MHA, and the impact of the Mental Capacity Act}

The Bournewood judgement, and subsequent developments in case law, changes in policy and the actions of CQC, have increased awareness of the situation of patients who may not have had the capacity to agree to admission or who may be prevented from discharging themselves. ${ }^{16}$ Such patients are more likely to be assessed for detention under the MHA than in the past.

In some services, staff who requested authorisation for patients to stay in hospital under the Deprivation of Liberty Safeguards reported long procedural delays in getting a visit from an assessor. This was often reported in wards caring for older people, many of whom have dementia. Staff in these services had turned increasingly to the MHA for authorisation of the care they were providing. Staff told us that, in past years, these groups of patients would have been less likely to be subject to the MHA, or any other legislative framework.

In all services, professionals reported a shift in culture to avoid de facto detention. This had led to a steady decline of informal patients over recent years; that may have sharpened following the 2014 Supreme Court ruling on the definition of

We asked for views about the noticeable increase in their service from 2013/14 to 2016/17 in detentions of people aged 65 and over. Ward managers explained they felt the Supreme Court case of Cheshire West (2014) was a possible explanation for this.

They told us of an increase from $60 \%$ people detained under the MHA to almost $100 \%$. One ward in the trust had increased from $15 \%$ to $85 \%$ in this period.

\section{Ward managers focus group} deprivation of liberty. ${ }^{17}$ Some areas reported that $80 \%$ of patients on acute wards are now detained and that on some older people's wards, every patient is detained.

\section{Increased involvement of the police in mental health admissions}

People told us that policy and legal changes to the MHA and the criminal justice system have raised awareness and understanding about the roles of police and mental health staff. For example, the increase in available health-based places of safety and greater local awareness of them may have led to an increased use of MHA powers by the police. There is now more collaboration between police and mental health services, including in the way that street triage services are provided. This increased recognition by the police of the mental health needs of people they have contact with, and the increased diversion of people with mental health problems from the criminal justice system, is welcome but could be contributing to the rising rates of detention. 


\section{Data reporting and data quality}

It is possible that some of the increase in the use of the MHA is due to features of the way that data about the use of the MHA are managed. Above, we described the impact that an increase in the number of patients with more than one admission in the course of the year might have on reported rates of detention.

It is also possible that some of the reported increase in the use of the MHA is an artefact caused by changes in the way in which local services manage and submit national data returns. There are two factors that might cause this - improvements in the completeness of data returns and duplicate reporting. One potential cause of the latter is the repeated reporting of an episode of detention when a detained patient is transferred from one ward to another or from one provider to another. For this to have an impact on the rise in rates of detention since 2010, this type of reporting error must be a new phenomenon or the number of patient transfers must have increased.

From January 2016, a new method of collecting has been introduced by NHS Digital to improve recording and reporting abilities. All mental health services are now required, by contract, to return accurate and timely data to the mental health services data set (MHSDS). Those with access to the MHSDS data are now able to report on pathways and provide equalities data, outcome reports and use these to advance our understanding of when and why the MHA is used for individual patients. However, the first year's report, published in October 2017, shows concerns with data coverage and data completeness that will limit our ability to use the data at national level. From the information that is available, we can see that improved and duplicate reporting may have had a significant contribution to the rise in detentions over recent years. NHS Digital estimate this could account for $12 \%$ of the rise based on information returned for $2016 / 17 .^{18}$

From our site visits, we found that the way in which local services record and collate information about the MHA varies widely. We did not find an example of a standard approach to recording, reporting, sharing, analysing or responding to MHA data locally. Some providers told us they had looked at data for the MHA as a result of our visit and this had led to them taking action. We also heard that:

- Local governance committees played an important role in monitoring information about the MHA and the impacts of service changes on the way the MHA is used. In some services there was a central committee that reported into the board and had a standing item on the board agenda. Information discussed in the committees varied but included numbers of detention, incidents and CQC's Mental Health Act Review reports.

- Clinical commissioning groups used MHA data in various ways. We found examples of where MHA data and joint aims to reduce the number of detentions had helped to secure funding for new schemes, crisis houses, new psychiatric decision units or increased investment in liaison services and primary care. However, there was not a standard approach to what data would be shared between commissioners and providers or what commissioning decisions would be made based on the local MHA activity.

- Multi-agency partnership meetings were in place, which frequently involved hospital staff, local authority representatives, ambulance services and police. This included sharing information and data between services to identify issues and take action to improve. The level of staff involved in these meetings varied. However, most areas told us they would report incidents or issues to executive directors when needed. 
- Approved Mental Health Professional (AMHP) services reported a wide variation in the way that they record or report data and how these are used by local health and local authority services. AMHP data help people, for example, to understand the full picture locally, showing how many assessments lead to detention, difficulties in delays as a result of bed management and problems accessing the right specialists or professionals. Without this information it would be difficult for governance committees to identify where service pressures are happening or for local NHS bodies to work in genuine partnership with local authorities to improve crisis support for patients.

- There is a need to improve specific data collection about pathways across all services. This includes positive practice in monitoring the redetention and readmission rates for services, and our concern that sites with rising detention levels were unclear from the available data whether they were due to a higher number of patients detained or more frequent detentions for the same patients. National bodies may be well-placed to provide this clarity. 


\section{What local learning can help national programmes reduce the use of the Mental Health Act?}

We asked this question during all of our visits. People were keen to discuss how their services operated, where the pressure points were and what could be done to address these locally and nationally. As expected, the responses between patient groups, service types and local agencies varied considerably. We were told that there is a need:

- To ensure that any local or national reduction in inpatient capacity has a corresponding increase in community services.

- To continue to develop alternatives to admission. This includes better access to services that deliver 'low level', long-term preventative work in the community and specialist care for groups such as older people and those with a learning disability, in addition to ensuring robust crisis response and acute home treatment in all areas of the country..

- For more initiatives, such as the street triage services, crisis cafes and crisis houses.

- For greater investment by commissioners in services that support patients following admission, assist in effective, timely discharge, and prevent readmission.

- To consider the benefits of services that are needs-based and offer a provision of housing and support services, for example step down services to support the discharge of patients who have no accommodation.

- For improved models of partnership working, including more integrated systems, support, training and investment with local authorities and acute and primary services.

- For a greater appreciation at national level of the impact on people's mental health of reducing social support and of austerity measures for local authorities, and of the impact of availability of services that support people outside of hospital.

- For a greater focus on recovery, goal-orientated detentions and outcomes-based commissioning in mental health to provide increased opportunities to reduce the use of the MHA.

- For strong service user networks, use of peer support workers and recovery colleges that promote co-production and collaborative reviews of services, treatments and support that might help to prevent readmissions.

- To strengthen local governance of the oversight of the MHA and to make efforts to improve reporting on uses of the MHA and to ensure robust local assurance about MHA reporting into MHSDS. This would result in better data quality, and therefore is useful in helping providers and commissioners to better plan to meet the needs of the population they serve.

- To improve the availability, quality and use of data on readmission rates to encourage collaborative working between providers, commissioners and national bodies, and to better understand the consequences of early discharge and potential for more frequent readmissions. 
- To improve IT systems and reporting, to enable a greater understanding of issues and pathways for patients.

\section{Conclusion and next steps}

Our review raises at least as many questions as it answers about the factors behind the rising rates of detention. All of the factors could be considered for further research or investigation. As a starting point, we propose the following set of hypotheses:

1. The rise in rate of detention since 2010 is in part due to the national data return being more complete or to an increase in duplicate returns.

2. More people are being detained on more than one occasion during a calendar year than was previously the case.

3. As there has been a shift towards caring for more people in the community, bed numbers have fallen and more people with severe mental health problems are living outside of a hospital setting and so are at risk of being detained.

4. Some people are being detained under the MHA who would previously not have been detained. There are two possible reasons for this. One is because clinicians are applying the criteria for detention differently to people with certain types of disorder (such as dementia or personality disorder). The other is because more people with mental health problems are coming to the attention of mental health care workers (for example, through schemes that divert people form the criminal justice system).

5. People who need admission and who would previously have agreed to informal admission are now refusing, and then being admitted as detained patients.

6. Admissions (some of which would be formal) that could in the past have been prevented are now not being prevented because less restrictive alternatives are not available.

7. There has been an increase in the total size of the population of England and an increase in the size of those sections of the population that are more at risk of detention.

8. There has been an increase in the prevalence of risk factors for detention, such as social exclusion and problematic drug and alcohol misuse.

The government has commissioned a panel to carry out an independent review of the Mental Health Act. They have prioritised carrying out their own examination of the causes of the rising use of detention and of the over-representation of people from Black and minority ethnic groups in the detained population. The specific attention that will be given to how we can improve our understanding and respond to the disproportionate use of the MHA for people from BME groups is particularly important and collaborative solutions will need to be developed across health and social care service. For the general rising use of the MHA, we hope that this report will help them to direct their enquiries. We believe that our findings support the view that changes to legislation might not in themselves have a major or immediate impact on rates of detention. That was not the case following the last reform of the MHA in 2007. 
We will support the work of the independent review of the Mental Health Act and continue to work in partnership with NHS England, NHS Improvement and the Department of Health to deliver the Five Year Forward View on Mental Health action plan. We will also support NHS Digital by using our regulatory powers to encourage providers to improve MHA data returns.

The MHA imposes a duty on CQC to monitor the MHA. CQC also assesses how well providers discharge their responsibilities under the MHA when it inspects providers. This includes whether the provider follows the guidance in the MHA Code of Practice. The findings of CQC inspection teams from their assessment of the MHA will influence our ratings of a provider, including our rating of how well-led the organisation is.

We would encourage mental health care providers and clinical commissioning groups to use our findings to help plan and review services. This report contains examples of what those in the sites we visited have done to monitor the impact of the MHA, counter the rising rates of detention and use information about the MHA to improve services for patients. We hope that local providers and commissioners find these helpful. 


\section{Acknowledgements}

We are grateful to everyone who contributed their time, support, advice and expertise to the focused work. We are grateful to the following trusts and independent providers for their collaboration in arranging and hosting our visits, and for making staff, patients and carers available to speak with us.

- 2gether NHS Foundation Trust

- Birmingham and Solihull Mental Health NHS Foundation Trust

- Cornwall Partnership NHS Foundation Trust

- Cygnet Healthcare

- East London NHS Foundation Trust
- Greater Manchester Mental Health NHS Foundation Trust

- Lancashire Care NHS Foundation Trust

- Leeds and York Partnership NHS Foundation Trust

- Northumberland Tyne and Wear NHS Foundation Trust
- St Andrews Healthcare

- Sheffield Health and Social Care NHS Foundation Trust

- South West London and St George's Mental Health NHS Trust

We also thank the local authority and Clinical Commissioning Group representatives from the following areas who met with us:

- Birmingham

- Blackburn with Darwen

- Blackpool

- Bolton

- Cornwall

- Gloucestershire

- Hackney

- Kingston
- Lancashire

- Leeds

- Luton and Bedfordshire

- Manchester

- Merton

- Newham

- Northamptonshire

- Richmond
- Salford

- Sheffield

- Sunderland

- Sutton

- Trafford

- Tower Hamlets

- Wandsworth

\section{Mental Health Act Advisory Group and Service User Reference Panel}

We are also very grateful to the members of our Mental Health Act Advisory Group and Service User Reference Panel (currently and previously detained patients), who have provided advice and guidance as we developed the scope and methodology, and considered the findings and how to use them to encourage improvements in services.

Our Advisory Group includes: Approved Mental Health Professional Leads Network, Association of Directors of Social Services, Black Mental Health UK, British Institute of Human Rights, Human Rights Implementation Centre, Mental Health Alliance, National Survivor User Network, NHS England, NHS Confederation, NHS Digital, NHS Providers (Foundation Trust Network), The Department of Health, and Royal College of Psychiatrists. 


\section{Appendix: The Mental Health Act and other legal frameworks}

The Mental Health Act 1983 (MHA) is the legal framework that allows mental health patients to be admitted to hospital, detained and treated against their wishes or cared for in the community under community treatment order or guardianship. This can only be done if they are putting their own or other people's health or safety at risk and they have, or appear to have, a mental disorder. It is critical to ensure that people can access appropriate treatment for their disorder, as without effective, responsive treatment as set out in the NICE/SCIE Quality standards and guidelines, people are more likely to relapse, be readmitted, and not have their rights upheld.

The MHA includes safeguards for people's rights when they are being detained or treated by professionals. It does this by providing rules and requirements for professionals to follow. The MHA Code of Practice is the statutory guidance for mental health professionals and services and explains how the MHA should be used in practice.

CQC's role is to check that patients' human rights are being protected and to look at how providers are applying the safeguards of the MHA and the guiding principles and standards of the Code of Practice, while they are being cared for or treated under the Mental Health Act in England.

\section{Differences between the legal frameworks for admission to mental health settings}

Mental Health Act: the legal framework for compulsorily treating people with mental health conditions where it is in the interests of their health or safety or the safety of others to do so, alongside the safeguards required to protect their rights while receiving appropriate treatment.

Mental Capacity Act: the legal framework for people who need to make decisions on behalf of someone else who lacks capacity. It sets out who can take decisions, in which situation and how they should go about this. This makes sure that they act in the person's best interest and empower people to make their own decisions wherever possible.

Deprivation of Liberty Safeguards (DoLS): the part of the Mental Capacity Act that provides safeguards that protect the rights of people who are deprived of their liberty so that they can be given necessary care or treatment.

Children Act 1989: the legal framework relating to children. Except for secure accommodation orders, the Children Act does not allow a child to be deprived of their liberty, and DoLS cannot be used for under 18s. Depending on the circumstances, authority to deprive the child of their liberty for purposes of assessment and treatment for mental disorder may come from the consent of a person with parental responsibility, by way of an application to court or the Mental Health Act. 


\section{References}

1. NHS Digital, Mental Health Act Statistics Annual Figures: 2016/17, October 2017

2. Department of Health, National Service Framework for Mental Health, September 1999

3. Department of Health, National Service Framework for Mental Health, September 1999

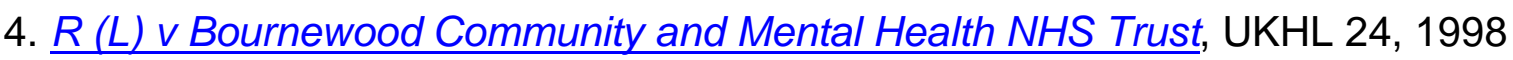

5. Mental Health Taskforce, The Five Year Forward View For Mental Health: A report from the independent Mental Health Taskforce to the NHS in England, February 2016

6. Crisp, N, Smith, G and Nicholson, K (Eds.) Old Problems, New Solutions - Improving Acute Psychiatric Care for Adults in England (The Commission on Acute Adult Psychiatric Care), 2016

7. Care Quality Commission, The State of Care in Mental Health Services 2014 to 2017, July 2017

8. Royal College of Psychiatrists, Progress on implementation of the report from the Commission on Acute Adult Psychiatric Care in England, one year after publication, February 2017

9. Mental Health Taskforce, The Five Year Forward View For Mental Health: A report from the independent Mental Health Taskforce to the NHS in England, February 2016

10. Crisp, N, Smith, G and Nicholson, K (Eds.) Old Problems, New Solutions Improving Acute Psychiatric Care for Adults in England (The Commission on Acute Adult Psychiatric Care), 2016

11. HM Government, Mental Health Crisis Care Concordant - Improving outcomes for people experiencing mental health crisis, February 2014

12. Care Quality Commission, 2017 Community Mental Health Survey: Statistical release, November 2017

13. https://improvement.nhs.uk/resources/red2green-improvement-tool/

14. Care Quality Commission, The State of Care in Mental Health Services 2014 to 2017, July 2017

15. Buchanan M, Patients sectioned because of pressure on beds, BBC News, 2 June 2014

16. $\underline{H L V U K}, \mathrm{ECHR} 471,2004$

17. Cheshire West and Chester Council V P, UKSC 19, 2014

18. NHS Digital, Mental Health Act Statistics, Annual Figures 2016/17, Experimental Statistics, October 2017 